\title{
Identification of Genes Related to the Early Stage of Angiotensin II-induced Acute Renal Injury by Microarray and Integrated Gene Network Analysis
}

\author{
Jing-Si Zhang ${ }^{a}$ Yun-Long Zhang ${ }^{a}$ Hong-Xia Wang ${ }^{b}$ Yun-Long Xia ${ }^{a}$ Lei Wang \\ Yi-Nong Jiang ${ }^{a}$ Hui-Hua Lia, ${ }^{a}$ Ying Liu ${ }^{a}$ \\ aDepartment of Cardiology, Institute of Cardiovascular Diseases, First Affiliated Hospital of Dalian \\ Medical University, Dalian; ${ }^{b}$ Beijing AnZhen Hospital the Key Laboratory of Remodeling-Related \\ Cardiovascular Diseases, Department of Physiology, Pathology and Pathophysiology, School of Basic \\ Medical Sciences, Department of Cardiology, Beijing Chaoyang Hospital, Capital Medical University, \\ Beijing; ' Center for Prevention and Control of Non-communicable Chronic Diseases, School of Public \\ Health, Dalian Medical University, Dalian, PR China
}

\section{Key Words}

Angiotensin II • Renal damage • Microarray • Gene expression profiles • Fabp1

\begin{abstract}
Background/Aims: Angiotensin II (Ang II) mediated signaling plays a key role in the development of chronic kidney damage that contributes to renal fibrosis. However, the gene expression changes regulated by Ang II in the early stage of acute renal injury remain unclear. Methods: C57BL/6 wild-type (WT) mice were injected with Ang II (1500 ng/kg/min) for 1,3 and 7 days. A time series analysis of microarrays was performed to evaluate Ang II-induced differentially gene expression in the kidneys. The data of gene expression in the kidney was further dissected by ANOVA analysis, gene expression profiles, gene network construction and quantitative real-time RT-PCR. Ang II-induced renal inflammation and fibrosis in mice were confirmed by pathological examination. Results: Our microarray data showed that a total of 1,511 differentially expressed genes were identified in the kidneys at 1, 3 and 7 days after Ang II infusion. These genes function in multiple biological processes, including response to stimuli, immune response, cell adhesion, metabolic process, kidney development, regulation of blood pressure, and ion transport, which may play critical roles in the pathobiology of Ang II-induced acute renal injury at the early stage. Furthermore, among these genes, 20 genes were further selected for final investigation. The dynamic gene network analysis demonstrated that fatty acid binding protein 1 (Fabp1) localized in the core of the network. Conclusions: Our data suggests that genes involved in lipid metabolic process, especially Fabp1, may play a central role in the development of Ang II-induced acute renal injury at the early stage.

J.-S. Zhang and Y.-L. Zhang contributed equally to this work.




\section{Introduction}

Hypertensive nephropathy is a major complication in hypertension, which causes disabilities and a high mortality rate in patients. Over time, hypertension can damage blood vessels and the tiny filtering units in the kidneys leading to renal injury, inflammation and fibrosis [1]. The mechanisms underlying the development of hypertensive nephropathy are extremely complex, however, the important role of the renin-angiotensin system (RAS) has been demonstrated. Angiotensin II (Ang II), the major peptide of the RAS, is involved in the pathogenesis of renal diseases via regulating inflammation and fibrosis [2,3]. There are two receptors AT1 and AT2 for Ang II. AT1 mediates most of actions of Ang II through enhancing proliferation, inflammation and fibrosis thereby contributing to chronic diseases, such as hypertension and renal damage [2, 3]. Importantly, AT1 receptor blockers (ARB) or angiotensin-converting enzyme inhibitors (ACEI) have been suggested to ameliorate the progression of hypertensive renal injury [4]. Of interest, it has also been shown that angiotensin blockade has clinical benefits for patients who have hypertension and renal disease [4].

Numerous studies have demonstrated that Ang II-mediated renal inflammation and fibrosis are mediated via multiple mechanisms. Transforming growth factor- $\beta 1$ (TGF- $\beta 1$ )/ Smad, Rho/Rho kinase and NF- $\mathrm{KB}$ are the main signaling pathways involved in Ang II-induced renal matrix production and recruitment of renal inflammatory cells $[2,3,5]$. Interestingly, inhibition of these pathways markedly suppresses the inflammatory and fibrotic processes $[3,6]$. Despite its critical role in the regulation of renal injury, inflammation and fibrosis, the mechanism of how Ang II causes the early acute renal injury at a molecular level remains to be elucidated. Recently, microarray analysis has been wildly used to investigate the global alterations of gene expression and identify genes that are important to cardiovascular and other diseases [7]. Therefore, microarray analysis may help us understand crucial factors that control the kidney damage and elucidate the precise molecular mechanisms of acute renal injury in Ang II-induced hypertension.

In this study, we investigated Ang II-mediated molecular events associated with the development of early renal injury by using microarray assay in a mouse model. Our results showed that after Ang II infusion at 1, 3 and 7 days, totally 1,511 genes with different expression variations were identified. These genes were then classified into 26 clusters based on expression pattern. Among these, five profiles (including No. 19, 21, 8, 22 and 6) of gene expression that contained a total of 814 genes were significant. Finally, the gene network analysis demonstrated that one gene Fabp1 localized in the core of the network, and may play a key role in the processes of Ang II-induced acute renal injury and fibrosis.

\section{Materials and Methods}

\section{Animals and Ang II administration}

Eight to ten-week-old C57BL/6 male mice were implanted with osmotic pumps (Alzet Model 1007D; Cupertino, CA,USA) and infused with Ang II at $1500 \mathrm{ng} / \mathrm{kg} / \mathrm{min}$ in Ringer's solution $(0.01 \mathrm{mmol} / \mathrm{L}$ acetic acid in saline) for 1, 3, and 7 days [8-10]. Systolic blood pressure was measured by the tail-cuff method before starting treatment and again every day after Ang II infusion [8-10]. All mice were kept at least $1 \mathrm{wk}$ at $22^{\circ} \mathrm{C}$ and 55\% relative humidity in a 12-h day/night lighting environment with free access to food and water. All procedures were performed in accordance with the Animal Care and Use Committee of Capital Medical University. The investigation conformed to the Guide for the Care and Use of Laboratory Animals published by the U.S. National Institutes of Health (NIH Publication No.85-23, revised 1996).

\section{Histology and immunohistochemistry}

The kidney tissues were fixed in phosphate-buffered $4 \%$ formalin ( $\mathrm{pH} 7.4$ ) for $24 \mathrm{~h}$ and then embedded in paraffin. The sections $(5 \mu \mathrm{m})$ were used for staining with H\&E, Masson's trichrome, and periodic acidSchiff stain (PAS) [8-10]. Tubular injury including brush-border loss, tubule dilatation, tubule necrosis, and tubule cast formation after Ang II injection was evaluated in PAS-stained sections. For immunohistochemical 
staining of Mac-2 (a marker for macrophages), tissue sections were deparaffinized and incubated with a Mac-2 primary antibody (Santa Cruz, CA, USA) as described [8-10]. The slides were evaluated and scored in a blinded fashion to the treatment by a renal pathologist. Images were viewed and captured using a Nikon Labophot 2 microscope (Nikon, Tokyo, Japan).

\section{RNA isolation and microarrays}

All mice were euthanized by an overdose of pentobarbital (100 mg/kg, i.p) on days 1, 3 and 7 of Ang II treatment. Total RNA was isolated with TRIzol (Invitrogen) from kidneys ( $\mathrm{n}=2$ per group) at each time point according to manufacturer's instructions. Gene expression profiling was performed using Affymetrix GeneChip Mouse Genome 430 2.0 Array according to the manufacturer's instructions (Affymetrix, Inc., Santa Clara, CA) [11, 12]. On the GeneChip Mouse Genome 4302.0 Array, over 45,000 probe sets analyze the expression level of over 39,000 transcripts and variants from over 34,000 well characterized mouse genes. The in detail gene expression data are also available at the GEO website under accession number GSE59437: http://www.ncbi.nlm.nih.gov/geo/.

\section{Analysis of microarray data}

A comprehensive bioinformatics analysis was used to enrich the dataset for genes that were most likely to be associated with the development of Ang II-induced acute renal injury, including Gene ontology (GO) analysis, pathway analysis, clustering analysis, and dynamic gene network analysis.

\section{Significant Differential Gene Analysis}

The random-variance model (RVM) F-test was applied to filter the differentially expressed genes for the saline-treated control and Ang II-treated group (at days 1, 3, and 7) because the RVM F-test can raise degrees of freedom effectively in the cases of small samples. After the significant analysis and the false discovery rate (FDR) analysis, we selected the differentially expressed genes according to the $P$-value threshold [13].

\section{Series test of cluster analysis}

The series test of cluster (STC) algorithm of gene expression dynamics was used to profile the gene expression time series and to identify the most probable set of clusters generating the observed time series. This method explicitly took into account the dynamic nature of the temporal gene expression profiles during clustering and identified the number of distinct clusters. We selected differential expression genes at a logical sequence according to RVM corrective ANOVA. In accordance with different signal density change tendency of genes under different situations, we identify a set of unique model expression tendencies. The raw expression values were converted into $\log _{2}$ ratio. Using a strategy for clustering short time-series gene expression data, we defined some unique profiles. The expression model profiles are related to the actual or the expected number of genes assigned to each model profile. Significant profiles have higher probability than expected by Fisher's exact test and multiple comparison test $[14,15]$.

\section{Gene ontology (GO) analysis}

GO analysis according to the Gene Ontology that is the key functional classification of NCBI, which can organize genes into hierarchical categories and uncover the gene regulatory network on the basis of biological process and molecular function. Specifically, two-side Fisher's exact test and $\chi^{2}$ test were used to classify the GO category, and FDR was calculated to correct the $P$-value, the smaller the FDR, the small the error in judging the $P$-value $[16,17]$.

\section{Pathway analysis}

Pathway analysis was used to find out the significant pathway of the differential genes according to KEGG, Biocarta and Reatome. We turn to the Fisher's exact test and $\chi^{2}$ test to select the significant pathway, and the threshold of significance was defined by $P$-value $(P<0.05)$ and FDR [18].

\section{Dynamic gene network analysis}

The normalized expression value of significant differential genes from the five most significant profiles, namely profiles $19,21,8,22$ and 6 , was used to build a co-expression network. In the network, 
Table 1. Primer sequences

\begin{tabular}{lll}
\hline Gene symbol & Forward primer $\left(5^{\prime}-3^{\prime}\right)$ & Reverse primer $\left(5^{\prime}-3^{\prime}\right)$ \\
\hline Fabp1 & TGAAGGCAATAGGTCTGCC & GTCATGGTCTCCAGTTCGCA \\
Fgl1 & GCTGGTGGTTTAACAGGTGTC & CAGGTGTACCACACAACACCA \\
Rgn & GGATACGGTCAGCAATCAAG & TCCCAGTTCAAAGCACAGAA \\
Ccdc69 & AAGCCTCGTTGCAGAACACC & TGGGCCAGGACTCCCATAAT \\
BB144871 & CTCCGGCCTTTCCTCTCTA & ATTTCAAGGTGACCACACAGG \\
Slc36a2 & CCCTACCTTGAGCTTGCCC & AATGTCTGGAACCCGGTTATG \\
OTTMUSG00000008561 & ACACATCCCAGCTACCCAAC & TTTCCCATGCGCTCATTCCT \\
Tmem30a & ATCGGCATCTTCGTCACCTC & ACACATTGCCCTCAAATGACTG \\
Tmem25 & TCACCAGCCGAGGTTTCATC & TGCCTCACCATCAAGCTCAG \\
Fads3 & ACAAGTGGCTGGTCATCGAG & CGTGGAAGGCATCCGTGG \\
Plagl1 & TCCGGCTAGGGTAGGTTGTT & CCAGAGCACAATAGCTGGGT \\
Ace & GGAAGCTCCTGGCGGATG & GTGTTTGGCGCCCAGTTG \\
Acy1 & AGACGTGGAGAGAGCAAGCAC & GCCAGGCACCACCTCTATTTT \\
Hk2 & AGTGGAACCCAGCTGTTTGA & ATGAAGTTGGCCAGGCATT \\
Hba-a1 & GGAAGACAAAAGCAACATCAAG & CATCAAAGTGAGGGAAGTAGGT \\
Bcl211 & GCTGCATTGTTCCCGTAGAG & GTTGGATGGCCACCTATCTG \\
Fkbp5 & AGATTCAGGCGTTATCCGTAGA & ATCAAATGTCCTTCCACCACAG \\
GAPDH & TCCCAGAGCTGAACGGGAAG & TCAGTGGCCCTCAGATGC \\
\hline
\end{tabular}

cycle nodes represent the genes, and edges between two nodes represent interactions between genes. All the nodes were marked with degree, which is defined as the link numbers one node has to the others. Genes with higher degrees occupied more central positions in the network and had a stronger capacity of modulating adjacent genes. In addition, k-core in graph theory was applied to describe the characteristics of the network. As network elements represent the ways in which genes may regulate other genes, large scale gene networks can be divided into certain subgraphs, and marked with different colors.

Validation of microarray data by qPCR analysis

Quantitative real-time PCR (qPCR) was used to verify the differential expression of 17 selected genes ( $n=4-6$ per group) that were detected by microarray. The primers used are listed in Table 1 . The first-strand cDNA was synthesized with moloney murine leukemia virus reverse transcriptase (Promega, Southampton, UK). qPCR was performed with an iCycler IQ system (Bio-Rad, USA) as described [8-10]. The cycling conditions consisted an initial, single cycle of $5 \mathrm{~min}$ at $95^{\circ} \mathrm{C}$, followed by 40 cycles of $30 \mathrm{sec}$ at $95^{\circ} \mathrm{C}, 30$ sec at $54^{\circ} \mathrm{C}, 15 \mathrm{sec}$ at $72^{\circ} \mathrm{C}$, and fluorescence acquisition at $83^{\circ} \mathrm{C}$ for $1 \mathrm{sec}$. The gene expression levels were quantified relative to the expression of GAPDH.

\section{Statistical analysis}

All values were presented as mean \pm S. E. M. Statistical differences between groups were analyzed by the non-parametric tests (Mann-Whitney) or by the parametric test one-way analysis of variance (ANOVA) followed by the Tukey-Kramer test for group differences. $P<0.05$ was considered significant.

\section{Results}

Angiotensin II induces renal inflammation and fibrosis in mice

Hypertensive mouse model was successfully induced by Ang II infusion. The blood pressure was markedly increased in a time-dependent manner (at days 1, 3 and 7) (Fig. 1A). Pathological examination indicated that continuous infusion of Ang II caused renal inflammation and fibrosis reflected by increased infiltration of Mac-2-positive macrophages and collagen deposition in the kidney tissues at days 3 and 7 (Fig. 1B). In contrast, PAS staining showed no significant effect of Ang II infusion on renal tubular injury (Fig. 1B).

\section{Genes detected by microarray assay in Ang II-infused kidneys}

To determine the gene expression changes in Ang II-induced acute renal injury at early stage, kidney tissues were taken from the mice at days 1, 3, and 7 after Ang II infusion, and a time series microarrays ( $\mathrm{n}=2$ per time point) were performed by using the Affymetrix probe dataset. Moreover, we found that total 1,511 genes that had a $P$-value and the false discovery 


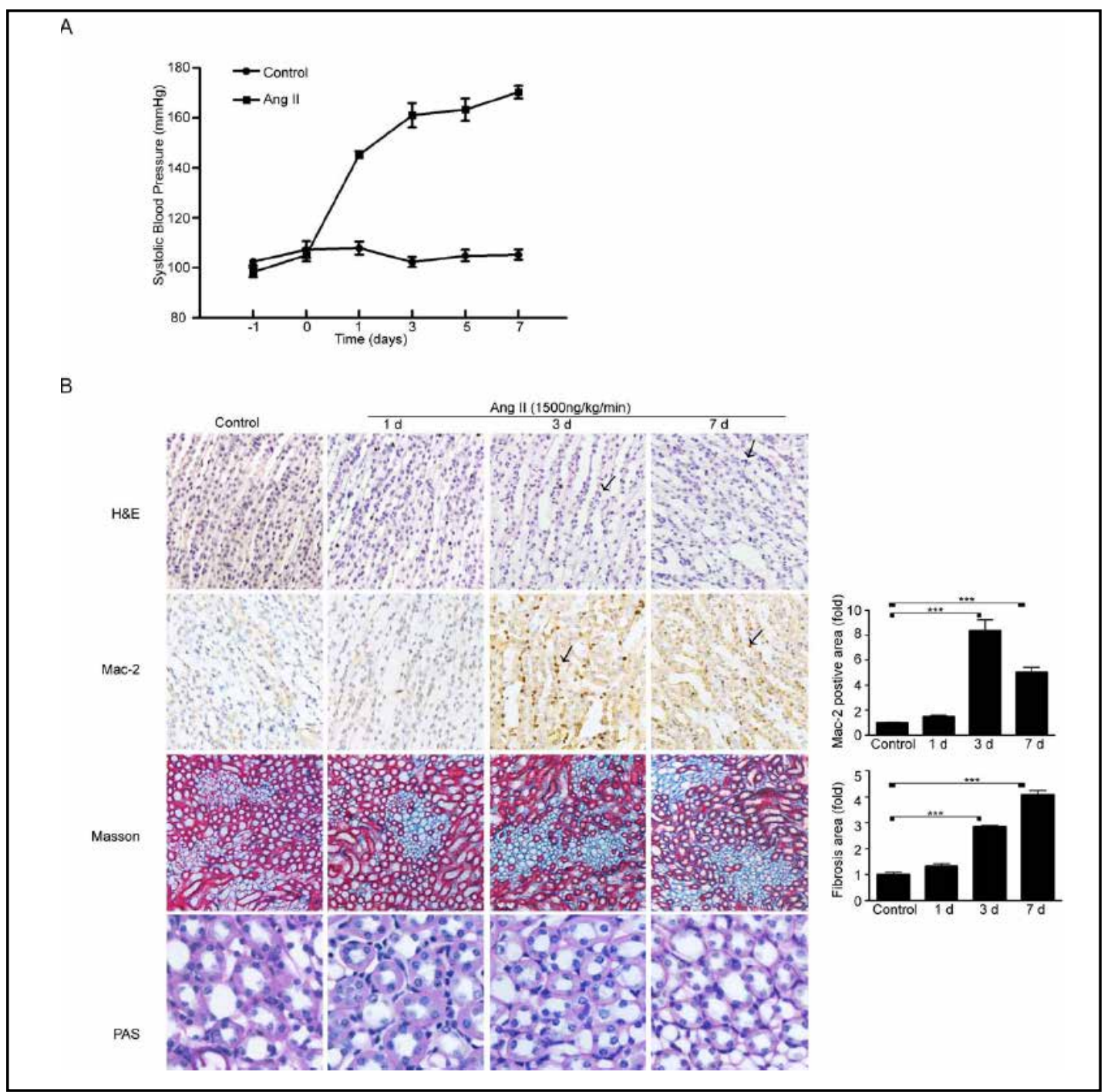

Fig. 1. Blood pressure and renal pathological examination in mouse model with acute renal injury induced by Ang II-infusion. (A) Wild-type mice were infused with Ang II (1500 ng/kg/min) for 1, 3 and 7 days. Systolic blood pressure was measured by the tail-cuff method ( $n=6$ in each group). (B) Pathological examination was performed with H\&E staining, immunohistocheimcal staining with anti-Mac-2 antibody, Masson's trichrome staining and PAS staining. Black arrows indicate Mac-2 positive cells. Data are shown as mean \pm S. E. M. (n=4 per group). ${ }^{* * *} P<0.0001$ vs. control.

rate (FDR) less than 0.05 were shown to be significantly expressed in Ang II-infused mice at least at one time point when compared to control $(P<0.05)$. Furthermore, when compared with probes in the saline-treated control, 640 probes significantly increased and 434 probes decreased in the $1 \mathrm{~d}$ group; 380 probes markedly increased and 277 probes decreased in the $3 \mathrm{~d}$ group, and 224 probes increased and 190 probes decreased in the $7 \mathrm{~d}$ group. These results indicate that by time after Ang II infusion the number of different gene expression in the kidneys was decreased.

To confirm the reliability of microarray data, qPCR analysis was performed for 10 genes at each time points, including Rgn, Ccdc69, Slc36a2, OTTMUSG00000008561, Tmem30a, Fads3, Ace, Acy1, Fkbp5 and Bcl2111, which were differentially expressed in our microarray assay. As shown in Fig. 2A-C, scatter plot graphs of gene expressions showed a significant correlation between microarray and qPCR results at days 1, 3, and 7 after Ang II infusion. 


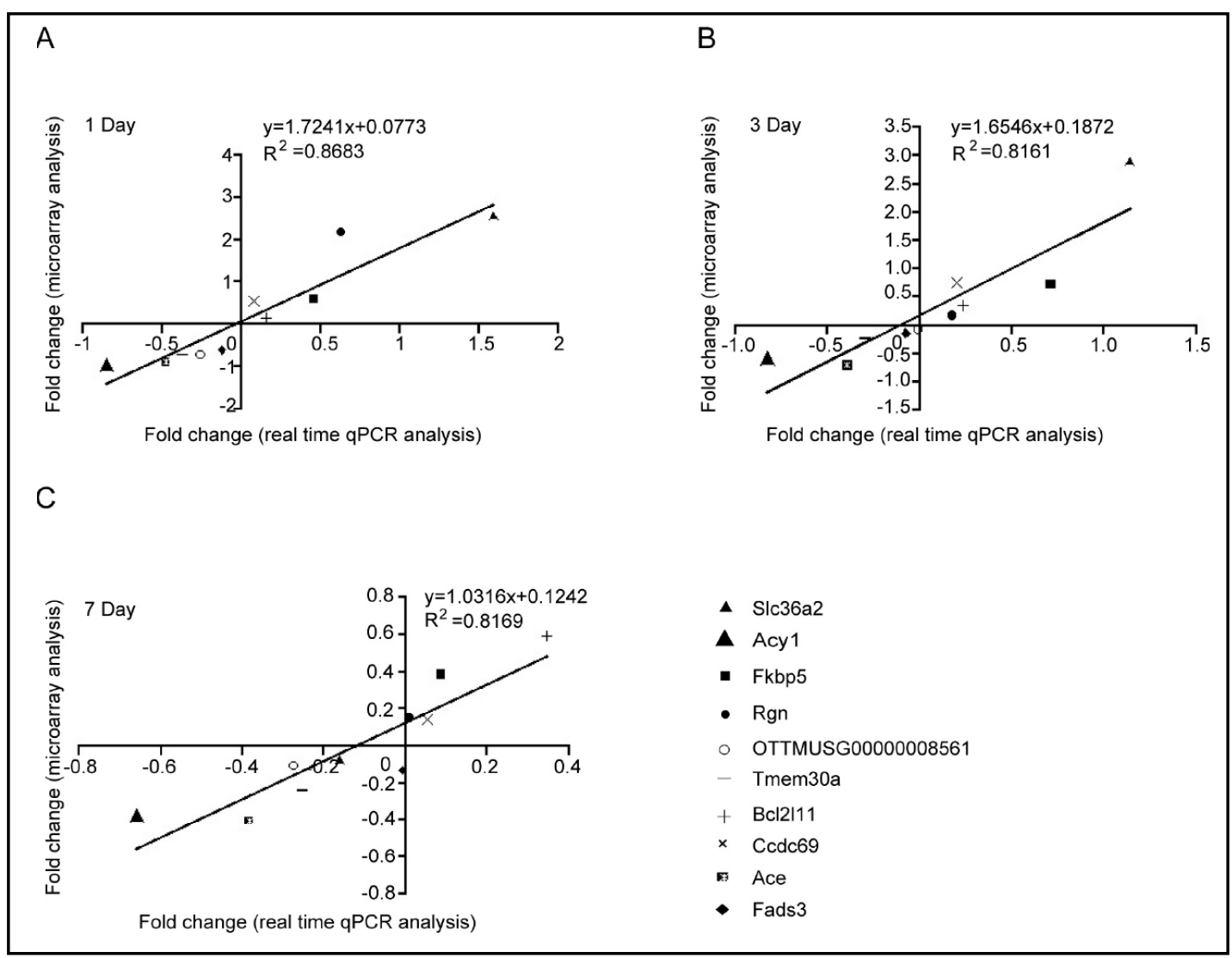

Fig. 2. Scatter plot of relative changes in gene expression as determined by microarray analysis and by qPCR analysis. To confirm the results obtained with the microarray, qPCR was performed for 10 genes at each time points, including Rgn, Ccdc69, Slc36a2, 0TTMUSG00000008561, Tmem30a, Fads3, Ace, Acy1, Fkbp5 and Bcl2l11. Quantification of gene expression by qPCR showed a significant correlation with microarray quantification.

Gene Ontology (GO) Analysis

GO analysis was used to analyze differentially expressed genes that had the main cellular functions during Ang II infusion. The most statistically significantly overrepresented GO terms $(P<0.001)$ were observed. As shown in Fig. 3, the high enrichment GO terms included response to stimuli (including drug, nutrient, glucocorticoid, lipopolysaccharide, hypoxia, peptide hormone, cAMP, mechanical, ethanol, cytokine, estradiol, hydrogen peroxide, cold, organic nitrogen and insulin), aging, organ regeneration, immune response, cell adhesion, metabolic process (including lipid, fatty acid, cholesterol and lipoprotein), kidney development, regulation of blood pressure, cellular processes (cell proliferation and differentiation), and ion transport, suggesting that these GO terms may play critical roles in the pathobiology of Ang II-induced early renal injury and fibrosis.

\section{Pathway analysis}

Biological pathway analysis yielded that 93 pathways $(P<0.05)$ were significantly modulated in the kidney tissues during Ang II infusion. Several key pathways associated with metabolic pathways (including PPAR, cytochrome P450, retinol, amino acids, fatty acid, nitrogen and lipid), immune/inflammatory process (including cytokine-cytokine receptor interaction, antigen processing and presentation, natural killer cell mediated cytotoxicity, chemokine signaling pathways, adipocytokine, leukocyte transendothelial migration and 


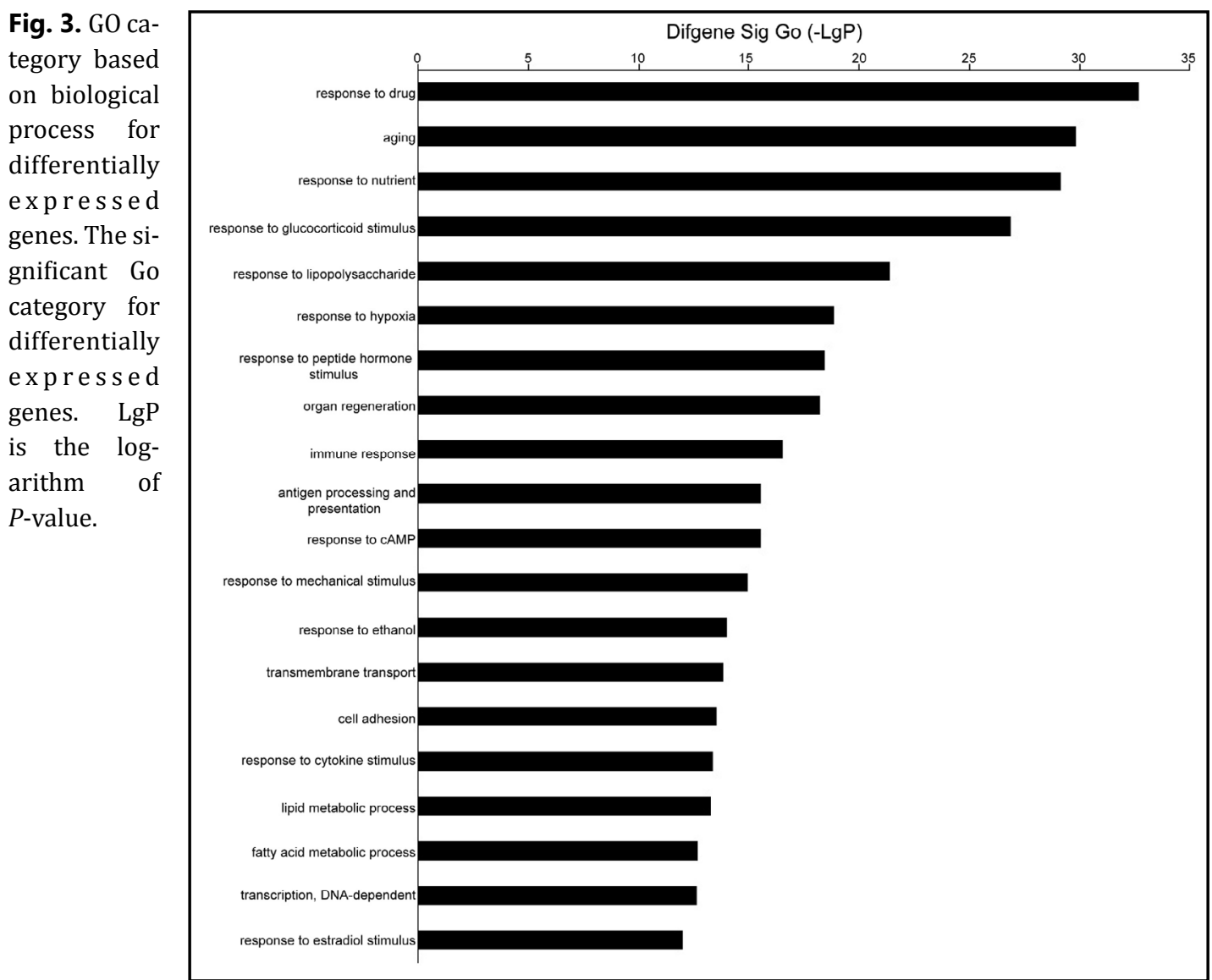

TLR), ECM-receptor interaction, rennin-angiotensin system, MAPK, mTOR, Jak-STAT and Wnt signaling pathways as well as apoptosis were significantly overrepresented (Fig. 4). The importance of metabolic pathways, immune response and ECM-receptor interaction, and rennin-angiotensin system at the early phase of Ang II infusion was highlighted and could be considered to be important for Ang II-induced renal inflammation and fibrosis.

\section{Cluster analysis of significant differential genes in Ang II-treated kidneys}

After hierarchical cluster analysis, a total 1,511 genes were classified into 26 profiles based on expression patterns, and each cluster has similar expression patterns after Ang II infusion (Fig. 5). Among these profiles, we identified 5 significant profiles (including No. $19,21,8,22$ and 6 ) of gene expression that contained a total of 814 genes. Gene expressions in profiles No. 19, 21 that are comprised of 275 and 176 genes respectively, were increased at early time points ( 1 or $3 \mathrm{~d}$ ) and then gradually decreased at later times $(7 \mathrm{~d})$; gene expressions in profile 22 were constantly increased at all time (1, 3, and $7 \mathrm{~d})$; while the genes in profiles No. 8 and 6 had opposite effects compared with genes in profiles No. 19, 21 (Fig. 6). Among these patterns, one most significant pattern was profile 19 according to ascending $P$-values. The profile No. 19 contained 275 genes that were the most abundant and exhibited increased expression at $1 \mathrm{~d}$ and decreased expression at days 3 and 7, after Ang II infusion. Thus, this significant profile is considered to be the potential main expression profile in our experiment, with the tendencies consistent with or opposite to that occurred during hypertension and acute renal injury induced by Ang II ( Fig. 1).

Dynamic Gene network analysis based on GO analysis

To further determine which gene or genes may play key roles in the early stage of acute renal injury induced by Ang II infusion, gene co-expression networks with the k-core algorithm were established from functional gene associations, and 814 genes selected in 5 
Fig. 4. KEGG pathway analysis for differentially expressed genes. The significant pathway for differentially expressed genes associated with renal injury. LgP is the logarithm of $P$-value.

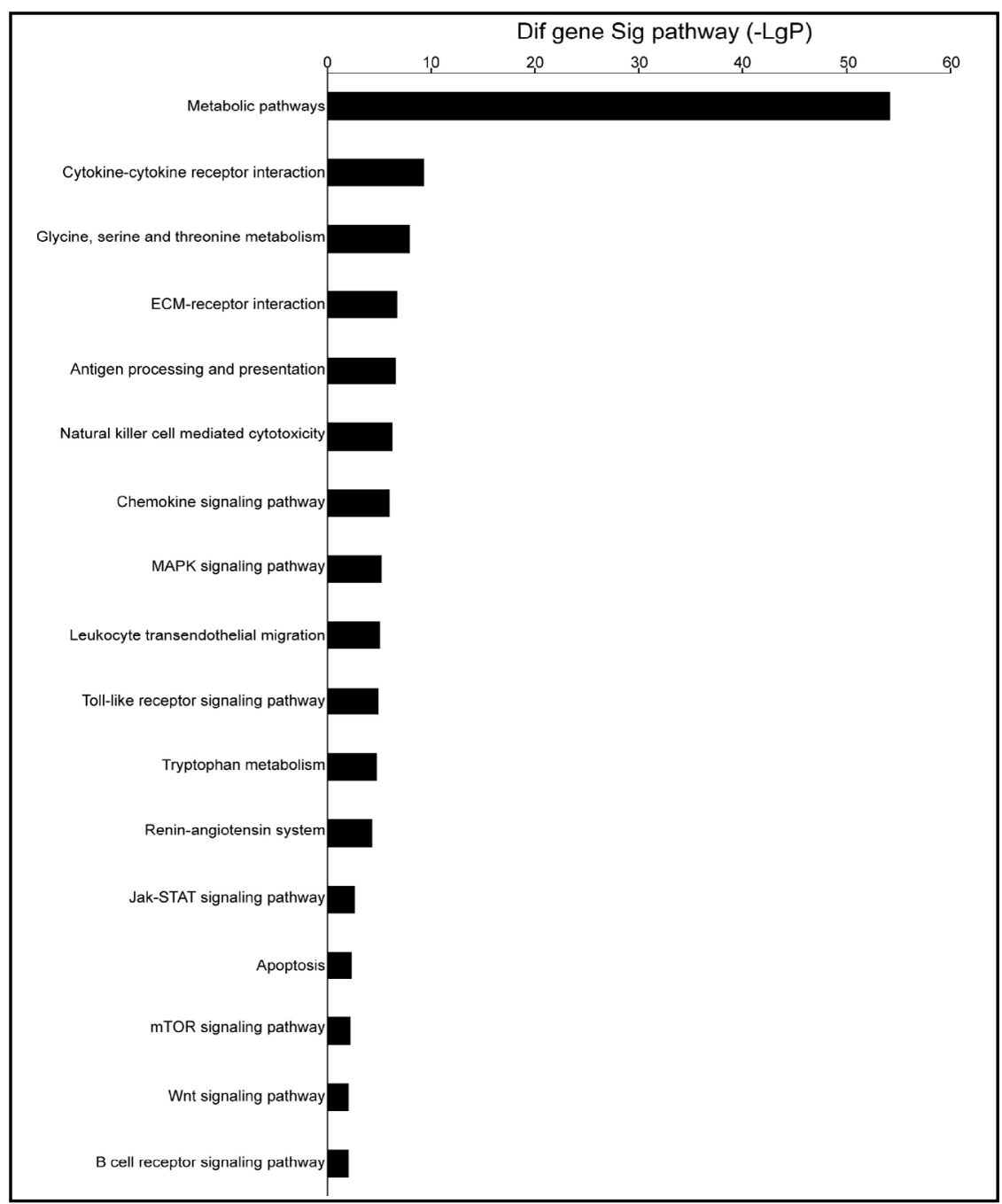

profiles were then analyzed. The correlations between genes were shown in Fig. 7. In the networks, cycle nodes represent genes, and edges between two nodes represent interactions between genes, which were quantified by degree. The k-core subnetwork with higher k-core level is considered to have a core status within a large scale gene network and is made up of differential 20 genes, which were from profile No. 19 (Table 2). Most of the genes were attributed to response to stimuli (including drug, nutrient, glucocorticoid, hypoxia), organ regeneration, metabolic process (including lipid, fatty acid, cholesterol, carbohydrate, steroid, amino acid), and ion transport. The core gene fatty acid binding protein 1 (Fabp1) appeared at the center of the large-scale networks and the $46 \mathrm{k}$-core subnetwork. It directly regulated 63 neighboring genes that interact according to their degrees. These interactions depended in large part on Fabp1 because the clustering coefficient of this gene was 0.85, which was lower than for other genes. In this network, Fabp1 was shown to play a direct role in the important processes of regulation of hydrolase activity, fatty acid beta-oxidation, intestinal absorption and cell proliferation. The result of Fabp1 up expression at 1 day may be related to development of early renal injury.

Verification of the gene expression by qPCR analysis

To further confirm the results from microarray results, qPCR was performed on the kidney tissues. As shown in Fig. 8, the relative expression levels of 17 differentially expressed genes from 5 significant profiles, including No. 19 (Fabp1, Fgl1, Rgn), No. 21 (Ccdc69, BB144871, Slc36a2), No.8 (OTTMUSG00000008561, Tmem30a, Tmem25, Fads3), 


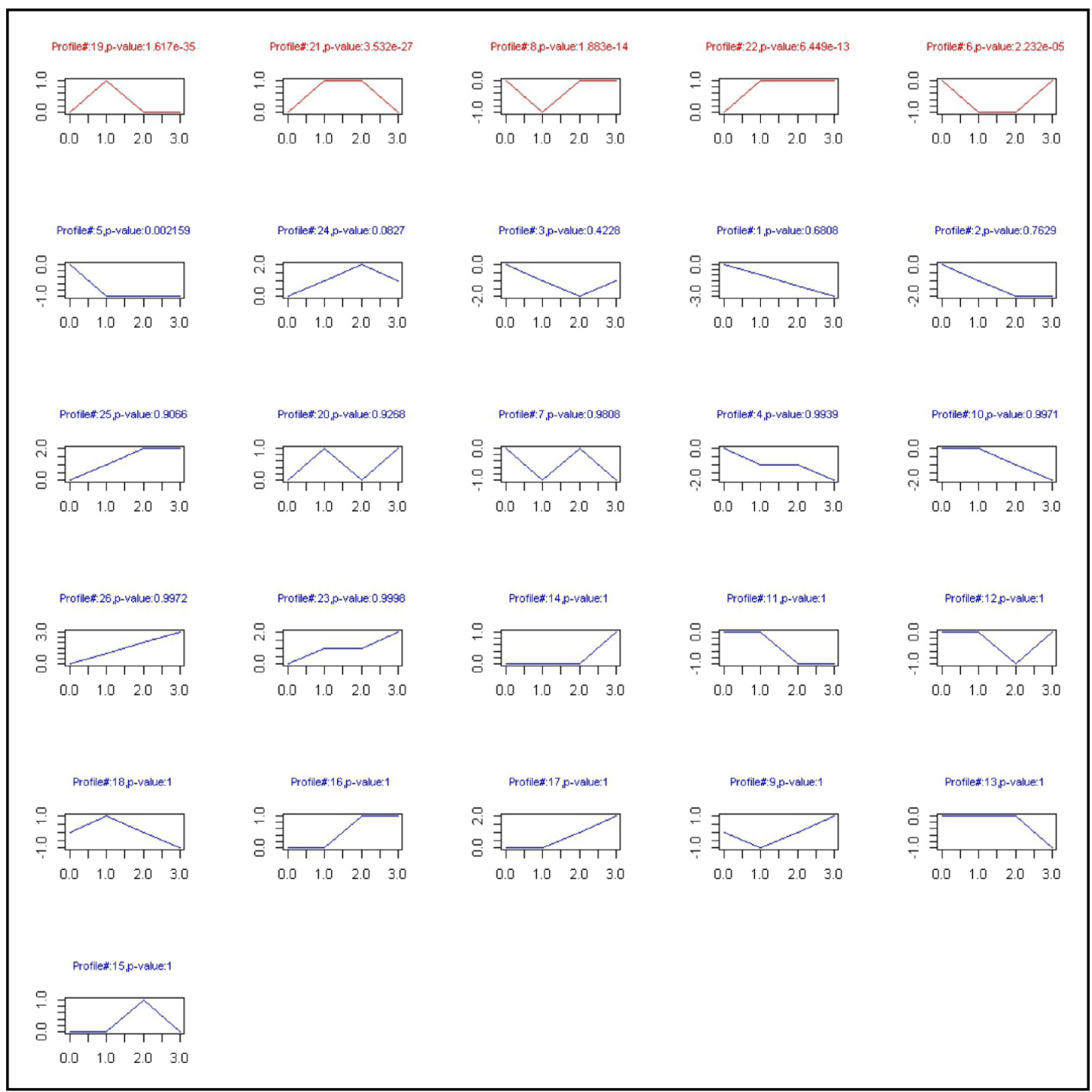

Fig. 5. The expression patterns of 1,511 genes by hierarchical cluster analysis. The expression patterns of 1,511 genes were classified into 26 profiles by hierarchical cluster analysis. Genes in the same box have a similar expression pattern. 5 significant profiles (including No. 19, 21, 8, 22 and 6) were identified $(P<0.001)$.

No. 22 (Plagl1), No. 6 (Ace, Acy1), and 4 non-significant profiles, including No.15 (Hk2), No.18 (Hba-a1), No.24 (Fkbp5) and No. 26 (Bcl2l11), were assayed. 8 genes Fabp1, Fgl1, Rgn, Ccdc69, BB144871, Slc36a2, Plagl1, and Hba-a1 were peaked at 1d, and decreased at 3 and $7 \mathrm{~d}$; six genes OTTMUSG00000008561, Tmem30a, Tmem25, Fads3, Ace, and Acy1 were decreased at all time points; two genes Fkbp5 and Hk2 increased at 1 and $3 \mathrm{~d}$, and returned to baseline at $7 \mathrm{~d}$; one gene Bcl2l11 was constantly increased at 1, 3, and 7 days, indicating that these data were good consistent between the two experiments (Fig. 6).

\section{Discussion}

Due to the high morbidity and mortality of renal disease, early diagnosis followed by early effective prevention is the key for prognosis improvement. So far, the genes related to the very early stage of acute renal injury and fibrosis induced by Ang II infusion remain 


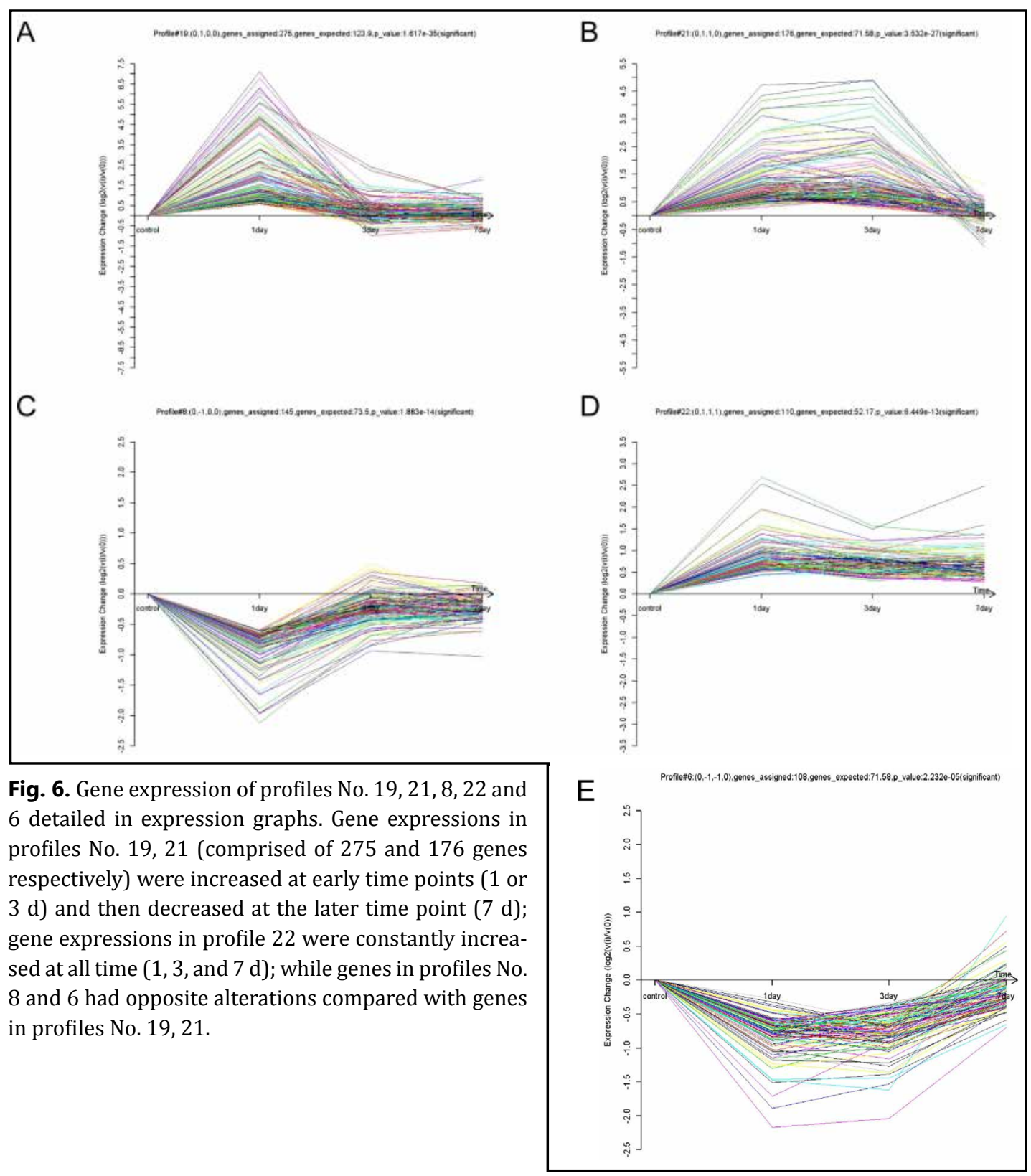

unknown. In the present study, we conducted time series microarrays and integrated gene network analysis to identify differentially expressed genes, GO terms, and KEGG pathways. Our results suggest that 1,511 genes were involved in multiple biological processes, including metabolic process, immune response, cell adhesion, kidney development, and regulation of blood pressure at early stage of acute renal injury induced by Ang II. Finally, our work further identified one key gene fatty acid binding protein 1 (Fabp1) that may play a key role in the development of acute renal damage and fibrosis. Taken together, this work identifies significant expressed genes and provides novel evidence for the molecular events associated with the development of early renal injury.

Renal fibrosis is a main pathological feature of progressive kidney disease [19]. It is characterized by accumulation of fibroblasts and excessive matrix proteins along with a loss of functioning nephrons [20]. Numerous studies have suggested that progressive renal fibrosis is mediated by various mediators, including growth factors, cytokines, metabolic toxins, and stress molecules through multiple signaling pathways [21]. Ang II participates 


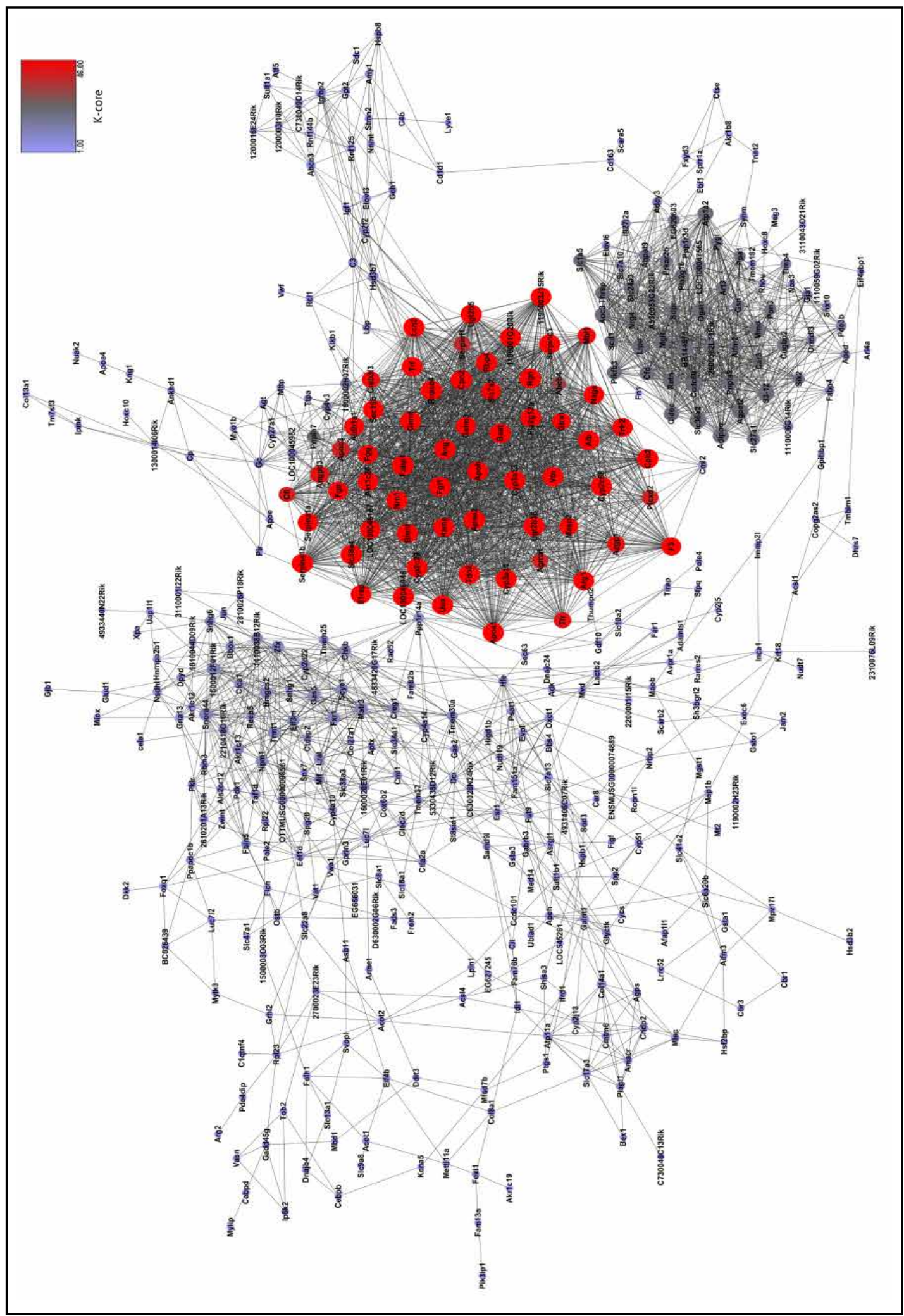

Fig. 7. Gene co-expression network. 814 genes from the 5 significant profiles (including No. 19, 21, 8, 22 and 6) were analyzed with k-core algorithm and gene co-expression networks were established. Cycle nodes represent genes, and edges between two nodes represent interactions between genes which were quantified by degree. The higher the degree is of a gene, the more central position it occupies in the network. 
in the pathogenesis of renal diseases via regulating two key processes inflammation and fibrosis [2]. Among them, Ang II plays a critical role in the pathogenesis of renal diseases through main AT1 and AT2 receptors. AT1 mediates most of the actions of Ang II, which activates several signaling pathways, including TGF- $\beta 1 /$ Smad, Rho/ Rho kinase, MAPK, mTOR, and Jak-STAT [22]. Recently, microarray assay has been wildly used to investigate the global changes of gene expression, and identify genes that are important to Ang II-induced response in smooth muscle cells, vascular, and kidney [23-25]. Despite its central role in the regulation of blood pressure and renal injury, the mechanism of Ang II actions in the kidney at a molecular level remains to be defined. In this study, we performed time series microarrays to identify significant differentially expressed genes, GO terms, and KEGG pathways. We found that a total 1,511 differentially expressed genes in the Ang II-treated kidney when compared to control. Of them, 1074 genes were markedly
Table 2. 20 genes identified by gene co-expression network with k-core algorithm

\begin{tabular}{llll}
\hline Gene symbol & Clustering Coefficient & Degree & k-core \\
\hline Fabp1 & 0.84946237 & 63 & 46 \\
Fgl1 & 0.87890005 & 62 & 46 \\
Rgn & 0.87890005 & 62 & 62 \\
Slc38a4 & 0.87890005 & 62 & 46 \\
Ang & 0.87149656 & 62 & 46 \\
Baat & 0.87043892 & 62 & 46 \\
Lcn2 & 0.86567953 & 62 & 46 \\
Cpn2 & 0.82072977 & 62 & 46 \\
Alb & 0.89672131 & 61 & 46 \\
Apoa1 & 0.89672131 & 61 & 46 \\
Apoa2 & 0.89672131 & 61 & 46 \\
Cyp2c29 & 0.89672131 & 61 & 46 \\
Cyp3a11 & 0.89672131 & 61 & 46 \\
Cyp3a13 & 0.89672131 & 61 & 46 \\
Hamp & 0.89672131 & 61 & 46 \\
LOC100046946 & 0.89672131 & 61 & 46 \\
Serpinc1 & 0.89672131 & 61 & 46 \\
Tdo2 & 0.89672131 & 61 & 46 \\
Ugt2b36 & 0.89672131 & 61 & 46 \\
Ugt2b5 & 0.89672131 & 61 & 46 \\
\hline
\end{tabular}
altered at day 1, and 657 genes at day 3. Interestingly, at day 2 of Ang II infusion, 805 genes were differentially expressed ( $>1.5$-fold), indicating that the data from Makhanova' study fits within the time-course of our results. Importantly, a lot of gene expression patterns in our results were similar with Makhanova's data [25]. Moreover, these genes participate in response to stimuli, organ regeneration, immune response, cell adhesion, metabolic process, kidney development, regulation of blood pressure, cellular processes, and ion transport. These observations were consistent with previous report by Makhanova, including response to stimuli, metabolism, transport, physiological processes, etc [25]. Moreover, the present data revealed that Ang II infusion significantly up-regulated metabolic pathways, immune/ inflammatory process, ECM-receptor interaction, rennin-angiotensin system, MAPK, mTOR, Jak-STAT and Wnt signaling pathways (Fig. 4), which are main mechanisms involved in Ang II-induced matrix regulation and inflammation [22, 26, 27]. Together, the analysis of Gene Ontology and pathway enrichment of these differentially expressed genes have provided novel insight into the molecular pathogenesis of acute renal injury and gene regulation induced by Ang II.

Fabp1 (also known as L-Fabp) is a 14-kDa organic anion-binding cytoplasmic protein produced mainly in the liver, and can be filtered via glomeruli because of its small molecular size and reabsorbed in proximal tubule epithelial cells like other small proteins [28]. Immunohistochemical analysis indicates that Fabp1 is exclusively localized to the cytoplasma of the proximal tubule cells [29]. Several studies suggest that Fabp1 is not expressed in both mouse and rat kidney in normal and pathophysiological conditions [30]. However, a recent study indicates that Fabp1 is expressed in the kidney [31]. Importantly, several clinical studies demonstrated the significance of urinary Fabp1 in kidney diseases. Fabp1 is superior to blood urea nitrogen (BUN) and urinary N-acetyl-beta-D-glucosaminidase (NAG) for early and accurate detection of acute tubular necrosis in different models of animal acute kidney injury. Thus, urinary Fabp1 is considered as a novel renal biomarker to predict the severity of kidney diseases, such as diabetic nephropathy [29]. Interestingly, Fabp1 has been reported to play an important role in the processes of fatty acid uptake, transport, 


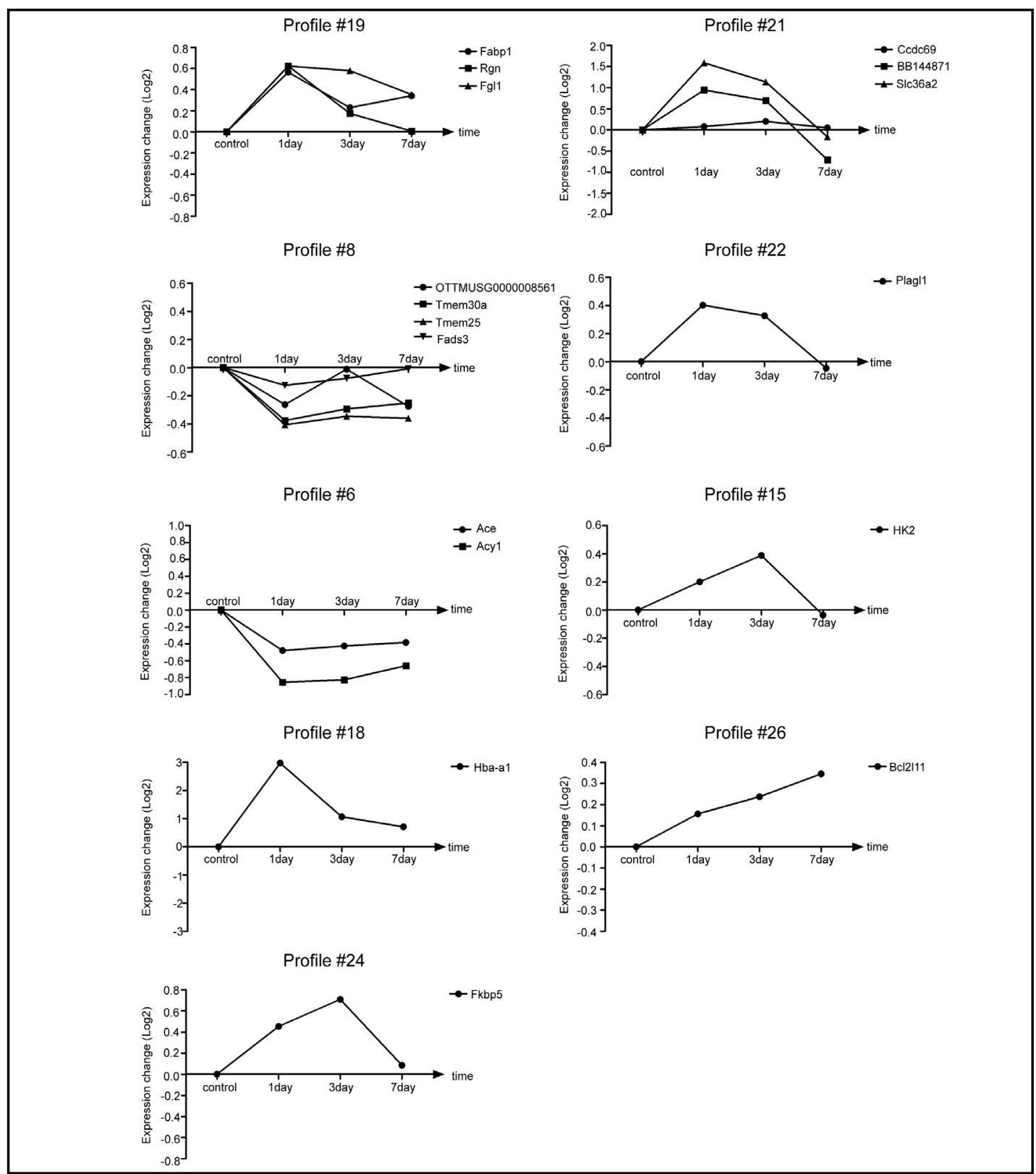

Fig. 8. Validation of microarray data by quantitative real-time PCR. Expression fold changes of 17 differentially expressed genes form both significant and non-significant profiles were verified by qPCR, including Fabp1, Fgl1, Rgn, Ccdc69, BB144871, Slc36a2,0TTMUSG00000008561,Tmem30a, Tmem25, Fads3, Plagl1, Ace, Acy1, Hk2, Hba-a1, Fkbp5, Bcl2l11. Data were presented as mean \pm S.E.M. (n=4-6 per group).

metabolism and fibrin clot formation. Fabp1 can bind selectively to free fatty acid (FFA) and is thought to be involved in energy production/metabolism in renal tubule cells [29]. Moreover, several recent studies suggest that Fabp1 plays a critical role in protecting renal injury through regulating oxidative stress, FFA metabolism. For example, human Fabp1 overexpression in proximal tubules reduces oxidative stress and tubulointerstitial damage induced by Ang II or unilateral ureteral obstruction [32, 33]. In the present study, cluster analysis indicated that Fabp 1 expression peaked at 1 day and decreased at day 3 and 7 after Ang II infusion. Gene network analysis further demonstrated that Fabp1 localized in the core of the gene network, indicating Fabp1 may contribute to initiation and development of acute renal damage. 
In this study, we investigated Ang II-mediated molecular events associated with the development of early renal injury by using microarray assay in a mouse model. We found that after Ang II infusion at 1, 3 and 7 days, 1511 genes were differently expressed in the kidney compared with control. Through an integrative analysis that combines changes in gene expression with gene function within a genetic network, we identified one gene Fabp1 that may be related to the very early stage of Ang II-induced renal injury and fibrosis.

\section{Acknowledgements}

This study was supported by grants from the 973 program (No. 2012CB517802), National Natural Science Foundation of China (81330003, 81025001), and Chang Jiang Scholar Program.

\section{References}

1 Mennuni S, Rubattu S, Pierelli G, Tocci G, Fofi C, Volpe M: Hypertension and kidneys: Unraveling complex molecular mechanisms underlying hypertensive renal damage. J Hum Hypertens 2014;28:74-79.

$>2$ Ruiz-Ortega M, Ruperez M, Esteban V, Rodriguez-Vita J, Sanchez-Lopez E, Carvajal G, Egido J: Angiotensin ii: A key factor in the inflammatory and fibrotic response in kidney diseases. Nephrol Dial Transplant 2006;21:16-20.

- 3 Esteban V, Lorenzo O, Ruperez M, Suzuki Y, Mezzano S, Blanco J, Kretzler M, Sugaya T, Egido J, Ruiz-Ortega M: Angiotensin ii, via at1 and at2 receptors and nf-kappab pathway, regulates the inflammatory response in unilateral ureteral obstruction. J Am Soc Nephrol 2004;15:1514-1529.

4 Remuzzi G, Perico N, Macia M, Ruggenenti P: The role of renin-angiotensin-aldosterone system in the progression of chronic kidney disease. Kidney Int Suppl 2005:S57-65.

5 Ruiz-Ortega M, Ruperez M, Lorenzo O, Esteban V, Blanco J, Mezzano S, Egido J: Angiotensin ii regulates the synthesis of proinflammatory cytokines and chemokines in the kidney. Kidney Int Suppl 2002:S12-22.

-6 Esteban V, Ruperez M, Vita JR, Lopez ES, Mezzano S, Plaza JJ, Egido J, Ruiz-Ortega M: Effect of simultaneous blockade of at 1 and at 2 receptors on the nfkappab pathway and renal inflammatory response. Kidney Int Suppl 2003:S33-38.

7 Supavekin S, Zhang W, Kucherlapati R, Kaskel FJ, Moore LC, Devarajan P: Differential gene expression following early renal ischemia/reperfusion. Kidney Int 2003;63:1714-1724.

-8 Jiang HM, Wang HX, Yang H, Zeng XJ, Tang CS, Du J, Li HH: Role for granulocyte colony stimulating factor in angiotensin ii-induced neutrophil recruitment and cardiac fibrosis in mice. Am J Hypertens 2013;26:12241233.

-9 Wang HX, Yang H, Han QY, Li N, Jiang X, Tian C, Du J, Li HH: Nadph oxidases mediate a cellular „memory“ of angiotensin ii stress in hypertensive cardiac hypertrophy. Free Radic Biol Med 2013;65:897-907.

10 Yang K, Zhang TP, Tian C, Jia LX, Du J, Li HH: Carboxyl terminus of heat shock protein 70-interacting protein inhibits angiotensin ii-induced cardiac remodeling. Am J Hypertens 2012;25:994-1001.

11 Zeng Y, Wang HX, Guo SB, Yang H, Zeng XJ, Fang Q, Tang CS, Du J, Li HH: Transcriptional effects of e3 ligase atrogin-1/mafbx on apoptosis, hypertrophy and inflammation in neonatal rat cardiomyocytes. PLoS One 2013;8:e53831.

12 Yang D, Zeng Y, Tian C, Liu J, Guo SB, Zheng YH, Li HH: Transcriptomic analysis of mild hypothermiadependent alterations during endothelial reperfusion injury. Cell Physiol Biochem 2010;25:605-614.

13 Wright GW, Simon RM: A random variance model for detection of differential gene expression in small microarray experiments. Bioinformatics 2003;19:2448-2455.

14 Ramoni MF, Sebastiani P, Kohane IS: Cluster analysis of gene expression dynamics. Proc Natl Acad Sci USA 2002;99:9121-9126.

15 Miller LD, Long PM, Wong L, Mukherjee S, McShane LM, Liu ET: Optimal gene expression analysis by microarrays. Cancer Cell 2002;2:353-361. 


\section{Cellular Physiology $\quad$ Cell Physiol Biochem 2014;34:1137-1151 and Biochemistry

-16 Ashburner M, Ball CA, Blake JA, Botstein D, Butler H, Cherry JM, Davis AP, Dolinski K, Dwight SS, Eppig JT, Harris MA, Hill DP, Issel-Tarver L, Kasarskis A, Lewis S, Matese JC, Richardson JE, Ringwald M, Rubin GM, Sherlock G: Gene ontology: Tool for the unification of biology. The gene ontology consortium. Nat Genet 2000;25:25-29.

17 Schlitt T, Palin K, Rung J, Dietmann S, Lappe M, Ukkonen E, Brazma A: From gene networks to gene function. Genome Res 2003;13:2568-2576.

18 Kanehisa M, Goto S, Kawashima S, Okuno Y, Hattori M: The kegg resource for deciphering the genome. Nucleic Acids Res 2004;32:D277-280.

19 Eddy AA: Molecular basis of renal fibrosis. Pediatr Nephrol 2000;15:290-301.

-20 Gao X, Wu G, Gu X, Fu L, Mei C: Kruppel-like factor 15 modulates renal interstitial fibrosis by erk/mapk and jnk/mapk pathways regulation. Kidney Blood Press Res 2013;37:631-640.

21 Lan HY: Diverse roles of tgf-beta/smads in renal fibrosis and inflammation. Int J Biol Sci 2011;7:1056-1067.

22 Hunyady L, Catt KJ: Pleiotropic at1 receptor signaling pathways mediating physiological and pathogenic actions of angiotensin ii. Mol Endocrinol 2006;20:953-970.

23 Jaffe IZ, Mendelsohn ME: Angiotensin ii and aldosterone regulate gene transcription via functional mineralocortocoid receptors in human coronary artery smooth muscle cells. Circ Res 2005;96:643-650.

24 Madhur MS, Lob HE, McCann LA, Iwakura Y, Blinder Y, Guzik TJ, Harrison DG: Interleukin 17 promotes angiotensin ii-induced hypertension and vascular dysfunction. Hypertension 2010;55:500-507.

25 Makhanova NA, Crowley SD, Griffiths RC, Coffman TM: Gene expression profiles linked to at1 angiotensin receptors in the kidney. Physiol Genomics 2010;42A:211-218.

-26 Huang Y, Noble NA, Zhang J, Xu C, Border WA: Renin-stimulated tgf-beta1 expression is regulated by a mitogen-activated protein kinase in mesangial cells. Kidney Int 2007;72:45-52.

27 Neilson EG: Mechanisms of disease: Fibroblasts--a new look at an old problem. Nat Clin Pract Nephrol 2006;2:101-108.

-28 Oyama Y, Takeda T, Hama H, Tanuma A, Iino N, Sato K, Kaseda R, Ma M, Yamamoto T, Fujii H, Kazama JJ, Odani S, Terada Y, Mizuta K, Gejyo F, Saito A: Evidence for megalin-mediated proximal tubular uptake of l-fabp, a carrier of potentially nephrotoxic molecules. Lab Invest 2005;85:522-531.

29 Noiri E, Doi K, Negishi K, Tanaka T, Hamasaki Y, Fujita T, Portilla D, Sugaya T: Urinary fatty acid-binding protein 1: An early predictive biomarker of kidney injury. Am J Physiol Renal Physiol 2009;296:F669-679.

-30 Sweetser DA, Lowe JB, Gordon JI: The nucleotide sequence of the rat liver fatty acid-binding protein gene. Evidence that exon 1 encodes an oligopeptide domain shared by a family of proteins which bind hydrophobic ligands. J Biol Chem 1986;261:5553-5561.

31 Schanstra JP, Bachvarova M, Neau E, Bascands JL, Bachvarov D: Gene expression profiling in the remnant kidney model of wild type and kinin b1 and b2 receptor knockout mice. Kidney Int 2007;72:442-454.

32 Ichikawa D, Kamijo-Ikemori A, Sugaya T, Yasuda T, Hoshino S, Igarashi-Migitaka J, Hirata K, Kimura K: Renal liver-type fatty acid binding protein attenuates angiotensin ii-induced renal injury. Hypertension 2012;60:973-980.

-33 Kamijo-Ikemori A, Sugaya T, Obama A, Hiroi J, Miura H, Watanabe M, Kumai T, Ohtani-Kaneko R, Hirata K, Kimura K: Liver-type fatty acid-binding protein attenuates renal injury induced by unilateral ureteral obstruction. Am J Pathol 2006;169:1107-1117. 